\title{
Regulation of an Open Access Essential Facility
}

\author{
By Axel Gautier $\dagger$ and Manipushpak Mitra $\ddagger$ \\ $\dagger C R E P P, H E C-U L G$ and CORE, UCL, Belgium $\ddagger E R U$-Indian Statistical Institute, Kolkata, India
}

Final version received 29 January 2007.

\begin{abstract}
A vertically integrated firm owns an essential input and operates on the downstream market. There is a potential entrant in the downstream market. Both firms use the same essential input. The regulator's objectives are (i) to ensure financing of the essential input and (ii) to generate competition in the downstream market. The regulatory mechanism grants non-discriminatory access of the essential facility to the entrant provided it pays a two-part tariff to the incumbent. The optimal mechanism generates inefficient entry. The inefficient entry captures the trade-off between market efficiency and infrastructure financing resulting from incomplete information and non-discriminatory access.
\end{abstract}

\section{INTRODUCTION}

In this paper, we are interested in the consequences of market liberalization in a regulated network-based industry. A common feature of such an industry is that the regulated incumbent firm owns an "essential facility", that is a facility that cannot be cheaply duplicated (e.g. long-distance electricity transmission grids, rail-tracks and stations, local wire line telecommunications, water supply networks). By 'market liberalization' we mean that a competitor can access the essential facility of the regulated incumbent if it chooses to do so.

It is well known that in the absence of market liberalization the regulator designs a welfare-maximizing contract for the (monopolist) incumbent. Under known (unknown) marginal cost of the incumbent, the welfare-maximizing contract specifies marginal cost pricing ('virtual' marginal cost pricing) and full reimbursement (type-contingent partial reimbursement) of the fixed cost of the essential facility with public funds (see Baron and Myerson 1982).

When there is a monopoly bottleneck in the production chain arising from the presence of the essential facility, competition and regulation are complements rather than substitutes (see World Bank 2002, Chapter 8). ${ }^{1}$ In these markets, allowing access of the incumbent's essential facility (that is, the bottleneck input) to competitors can help (a) in achieving welfare-enhancing competition for the final output and (b) in financing the cost of maintenance of the essential facility. In this paper we provide a theoretical model to address this problem of regulating a market with essential facility by allowing for endogenous market structure on the downstream market and assuming incomplete information.

In the theory of access pricing proposed by Laffont and Tirole (1994), the market structure is assumed to be exogenous and the access charges aim at maximizing the total consumer surplus, taking the number and the type of competitors as given. In our main problem, the regulator specifies the access charges without knowing the entrant's cost conditions and then the entry decision is taken. Hence the market structure is endogenous, and depends on both the regulatory environment and the entrant's cost conditions. Auriol and Laffont (1993) consider the problem of firms with unknown cost competing ex ante for a market. In their problem the market structure, ex post, is part of the regulatory mechanism and the regulator can allow the firms to operate as a duopoly

(C) The London School of Economics and Political Science 2007 
in order to reduce information rent. Hence the mechanism in Auriol and Laffont (1993) completely regulates the market. A similar problem was analysed by Dana and Spier (1994), Jehiel and Moldovanu (2004) and Mougeot and Naegelen (2005). We depart from these frameworks and analyse the situation in which it is not possible for the regulator to regulate the activities of the potential entrant. Hence the main features of the endogenous market structure of our problem are (a) the absence of ex ante competition for the market, (b) the presence of ex post competition on the market and (c) endogenous entry decisions.

In our problem we assume that the regulator cannot extract the entrant's cost information and hence allows the entrant to use the essential facility on an open access basis, provided the entrant pays the incumbent some specified price for its use. 'Open access' means that any competitor that meets some pre-specified requirements (e.g. technical, safety or financial fitness requirements) can have access to the essential input on a non-discriminatory basis. For example, in the European Union, railway undertakings need to apply for a licence and a safety certificate delivered by the member states to provide rail services. Granting a non-discriminatory access to the essential input is quite common in practice. ${ }^{2}$ It is also quite common to have an asymmetric regulatory regime between the incumbent and the entrant(s). The entrants are often free to pick and choose the market in which to operate and the consumers they want to serve while the incumbent is forced to serve all consumers (e.g. universal service obligations).

Our problem integrates these two features: open and non-discriminatory access to the essential input, and different regulatory regimes for the incumbent and the entrant. We call this sort of regulation an 'indirect' one because the regulator does not regulate the entrant but simply allows open access of the essential facility provided the entrant pays a two-part tariff to the incumbent. This two-part tariff is contingent only on the known marginal cost (or known distribution of the marginal cost) of the incumbent. We use a two-part tariff for pricing access to the network because in our framework the potential entrant has market power. The variable part of the tariff is used to countervail the entrant's market power and the fixed part is used to collect revenue for financing the network's costs. The access charge paid by the entrant helps the regulator to reduce (at least partially) the burden of recovering the cost of the essential input.

We also address the question of the efficiency of entry. At this point one needs to distinguish between efficient entry and efficient market structure. Entry is said to be efficient (in our type of market liberalization framework) if the competitor can, by entering the market, increase the welfare over that obtaining in the regulated monopoly situation. It is obvious that welfare can increase with entry only if the entrant's marginal cost is below that of the incumbent. Thus, when the market is open to a rival firm, efficient entry means that the entrant enters the market if and only if it has a lower marginal cost than the incumbent. An efficient market structure is more of an efficient market design issue. It corresponds to the first-best situation where the regulator gives monopoly rights to the firm with the lower marginal cost. Under the first-best analysis it is implicit that the regulator knows the marginal costs of the incumbent and the entrant, the regulator dictates the production level of the two firms, and the regulator has the ability to shut down a firm, specifically the one, with higher marginal cost. The papers of Auriol and Laffont (1993) and Dana and Spier (1994) focus on such a market design problem. Our framework deals with an open-access setup with no direct regulation of the entrant. In our setting entry is typically inefficient; that is, either a cost-efficient entrant 
stays out of the market, or a cost-inefficient entrant enters the market (see Baumol et al. 1982 and Armstrong 2001).

Our indirect regulatory mechanism prescribes (i) above-marginal-cost pricing if entry does not occur and below-marginal-cost pricing if entry takes place, (ii) a positive lumpsum entry fee to be paid by the entrant to the incumbent, (iii) a per-unit subsidy to be paid by the incumbent to the entrant, (iv) a public subsidy to (partially) finance the infrastructure cost, (v) a reduced share of public transfer for infrastructure financing relative to the case of a regulated monopolist, and (vi) no cross-subsidization of the incumbent's network costs by the incumbent's profit on the downstream market. Under this indirect regulatory regime, it is possible that a potential entrant, more cost-efficient than the regulated incumbent, stays out of the market.

We also consider the problem of indirect regulation when the cost of the incumbent and entrant are unknown. In this context, the regulatory mechanism provides an information rent to the more efficient incumbent firm. Given that these rents are socially costly, the regulator partially substitutes the incumbent's production by the entrant's production. Hence there is more entry. Moreover, inefficient entry can occur in both directions; that is, it is possible that an entrant less efficient than the incumbent enters the downstream market, and it is also possible that an entrant more efficient than the incumbent stays out of the market.

The paper is organized in the following way. We conclude this section by comparing our results with some of the existing literature. In Section I we introduce our model, in Section II we provide our main results, and we conclude our analysis in Section III. All proofs are relegated to the Appendix.

\section{Related literature}

One can compare the results of our paper with other papers dealing with the problem of regulation with an endogenous market structure. Caillaud and Tirole (2004) consider the problem of infrastructure financing under asymmetric information. As in our problem, an open access policy raises welfare in their paper, but since competition reduces profit the project can become non-viable if one allows open access. However, in their model a monopoly franchise on the downstream market is granted to the incumbent in situations where competition may have a high future profitability. This pattern results from the common value environment. Both social welfare and profit increases with market profitability, and hence extraction of incumbent's private information is impossible without the granting of a monopoly franchise. The regulator and the incumbent firm are in a situation of non-responsiveness where efficiency and incentives conflict (see Guesnerie and Laffont 1984).

Caillaud (1990) considers the problem of regulating an incumbent firm facing entry possibility on the downstream market. As in our own setting, unregulated competitors can enter the downstream market after the regulator has specified the regulatory mechanism; but competitors bypass the essential input of the incumbent and supply the service on the downstream market using an alternate technology. The regulator then has fewer instruments with which to influence the entry decision and cannot credibly deter entry by setting large access fees. In Caillaud's framework there is a competitive fringe of entrants on the market, and the regulator shuts down the incumbent if the competitors are expected to be more efficient than the incumbent. This is not possible in our framework, since shut down implies that the market is left to an unregulated monopolist.

(C) The London School of Economics and Political Science 2007 
Finally, Lewis and Sappington (1999) and De Fraja (1999) study, as we do, an access pricing problem in the context of asymmetric information. Lewis and Sappington assume that the regulator does not know the entrant's cost and that entry always takes place. So in their paper the market structure is given. De Fraja (1999) assumes that the entrant's cost is known and that the incumbent's cost is unknown. In his framework inefficient entry is observed: it is optimal to let a less efficient competitor operate on the downstream market because, owing to the unknown cost of the incumbent, it is always better partially to substitute the incumbent's output with that of the entrant.

\section{THE MODEL}

A regulated incumbent firm provides services to the final consumers. To provide these services, the incumbent uses its essential facility (network) as input. There is a potential entrant on the downstream market. To operate on the downstream market, both the incumbent and the entrant use the same essential input. The entrant can use the incumbent's essential input provided it pays the incumbent an appropriate access charge. The incumbent also receives public subsidies from the regulator to finance the network. The regulator (or public authority) specifies the amount of services that the incumbent should supply to the consumers. The regulator also specifies the amount of access charge that the incumbent should receive from the entrant if it decides to enter the downstream market. The access charge in our problem is a two-part tariff. The regulatory mechanism is observable, and hence the entry decision of the potential entrant depends on the quantity to be produced by the incumbent and the two-part tariff that the entrant has to pay to the incumbent if it decides to enter the downstream market. Figure 1 depicts the timing of the events.

The downstream market demand function is $P(Q)=a-b Q$, where $a>0, b>0, Q$ is the quantity demanded and $P(Q)$ is the market-clearing price corresponding to $Q$. The vertically integrated incumbent uses its essential facility and provides services to the final consumers. The (known) fixed cost of the infrastructure is $c>0$. For simplicity, we assume that there is no cost of using the infrastructure. The incumbent can supply services at a constant marginal cost $\theta \in[\underline{\theta}, \bar{\theta}]$. The total cost of the integrated firm (or incumbent), when it supplies $q_{i}$ units, is $C\left(q_{i}, \theta\right)=\theta q_{i}+c$. We will consider the possibility of both known and unknown cost. When the cost is unknown, we need the following assumptions for our analysis.

1. The marginal cost has a continuous density $f(\cdot)$ and $f(\theta)>0$ for all $\theta \in[\underline{\theta}, \bar{\theta}]$.

2. The distribution satisfies the hazard rate condition: $L(\theta)=F(\theta) / f(\theta)$ is increasing in $\theta$. Here $F(\theta)=\int_{x=\theta}^{x=\theta} f(x) \mathrm{d} x$ is the distribution function.

The potential entrant can produce an amount $q_{e}$ at a marginal cost of $\phi \in[\underline{\theta}, \bar{\theta}]$.

Throughout the paper, we assume that the marginal cost of the entrant is unknown and that it has a continuous distribution function $G(\phi)$ and a density function $g(\phi)$ where $g(\phi)>0$ for all $\phi \in[\underline{\theta}, \bar{\theta}]$. However, for closed-form solutions of our problem we will

$\begin{aligned} & \text { The regulator designs } \\ & \text { the regulatory mechanism }\end{aligned}$
$\begin{aligned} & \text { Firms learn on the downstream market } \\ & \text { their costs privately time }\end{aligned}$
$\begin{aligned} & \text { The potential entrant } \\ & \text { decides on entry }\end{aligned}$

FIGURE 1. The timing of the events. 
assume that the marginal cost of the entrant follows a uniform distribution; that is, $g(\phi)=1 / \Delta$ for all $\phi \in[\underline{\theta}, \bar{\theta}]$ where $\Delta \equiv \bar{\theta}-\underline{\theta}$. We also assume that the marginal cost of the entrant is independent of the marginal cost of the incumbent. When correlation between the incumbent's and the entrant's cost is positive, the regulatory problem is in general non-concave, hence intractable (see Caillaud 1990). With independence, the problem may be non-concave too, but a solution is possible. If the potential entrant decides to enter the market, it has to pay a two-part access charge: a fixed fee $A$ and a per-unit fee $\alpha$. The amounts of $A$ and $\alpha$ are specified by the regulator.

The regulator maximizes a weighted sum of consumer and producer surplus net of transfer: $W=S(Q)-P(Q) Q+\beta\left\{\pi_{i}+\pi_{e}\right\}-t$, where, $S(Q)=\int_{0}^{Q} P(x) \mathrm{d} x, \pi_{i}$ is the profit of the incumbent, $\pi_{e}$ is the profit of the entrant, $t$ is the amount of transfer paid by the regulator to the incumbent and $\beta \in[0,1]$ is the welfare weight attached to the producers' surplus. In this context, the transfer $t$ is meant to finance part of the essential input cost. We assume that there exists a non-distortionary tax system and hence the shadow cost of public funding is equal to zero. Adding a non-zero shadow cost of public funding adds nothing substantial to the analysis but more distortions. The results with non-zero shadow cost are qualitatively similar to what we get in this paper without having such a cost. Hence the regulator has four instruments at her disposal: the quantity of the incumbent $q_{i}$, the transfer $t$, the fixed fee $A$ and the per-unit fee $\alpha$.

There are two stages to our problem. In the first stage the regulator offers a regulatory mechanism to the incumbent. The regulatory mechanism $M_{i r}=\left\langle q_{i}(\cdot), A(\cdot)\right.$ $\alpha(\cdot), t(\cdot)\rangle$ specifies a quantity-fixed access charge - per-unit access charge - transfer quadruple that depends on the marginal cost of the incumbent. In particular, $q_{i}:[\underline{\theta}, \bar{\theta}] \rightarrow \mathbf{R}_{+}, A:[\underline{\theta}, \bar{\theta}] \rightarrow \mathbf{R}, \alpha:[\underline{\theta}, \bar{\theta}] \rightarrow \mathbf{R}$ and $t:[\underline{\theta}, \bar{\theta}] \rightarrow \mathbf{R}^{3}$. We restrict attention to continuous mechanisms only. Given the regulatory mechanism, in stage 2 , the potential entrant takes its entry decision. The entrant observes the regulatory mechanism before making its entry and quantity decisions (Stackelberg follower). The price is then set to equate demand and supply. The Stackelberg structure of the quantity game reflects the dominant position of the incumbent in the downstream market. We call this an indirect regulation mechanism.

Before going into the regulator's first-stage problem of designing the indirect regulatory mechanism, we conclude this section with two subsections. In the first we analyse the optimal second-stage entry decision of the entrant. In the second subsection we discuss the first-best mechanism where the marginal costs of the incumbent and the entrant are common knowledge and where it is possible to completely regulate both the incumbent and the entrant.

\section{Entrant's entry decision}

In our model the entry decision is taken after the regulator has designed the regulatory mechanism. Therefore, even though the entrant is not directly regulated, the regulatory mechanism (except the transfer received by the incumbent) affects the entry decision and the quantity supplied by the entrant. The entrant's maximization problem in stage 2 is to select $\left.q_{e}(\theta, \phi)=\max _{q_{e}}\left\{P\left(q_{e}+q_{i}(\theta)\right)-\alpha(\theta)-\phi\right\} q_{e}-A(\theta)\right\}$. Assuming that entry takes place, the solution to this problem is

$$
q_{e}(\theta, \phi)=\frac{a-\alpha(\theta)-\phi}{2 b}-\frac{q_{i}(\theta)}{2}
$$


The profit of an entrant active in the downstream market is

$$
\bar{\pi}_{e}(\theta, \phi)=\frac{\left\{a-\alpha(\theta)-\phi-b q_{i}(\theta)\right\}^{2}}{4 b}-A(\theta) .
$$

It is now obvious from (2) that the potential entrant enters the downstream market if $\phi \in[\underline{\theta}, K(\theta))$ where $K(\theta)=P\left(q_{i}(\theta)\right)-\alpha(\theta)-2 y(\theta)$ and $y(\theta)=\sqrt{b A(\theta)}$. Moreover, the profit of the entrant, with marginal cost $\phi \in[\underline{\theta}, \bar{\theta}]$, is

$$
\pi_{e}(\theta, \phi)=\left\{\begin{array}{ll}
0 & \text { if } \phi \geqslant K(\theta) \\
\{P(Q(\theta, \phi))-\alpha(\theta)-\phi\} q_{e}(\theta, \phi)-A(\theta) & \text { if } \phi<K(\theta)
\end{array},\right.
$$

where $Q(\theta, \phi)=q_{i}(\theta)+q_{e}(\theta, \phi)$. Given the second-stage optimization of the entrant, the ex post profit of the incumbent with marginal cost $\theta \in[\underline{\theta}, \bar{\theta}]$, is

$$
\pi_{i}(\theta, \phi)=\left\{\begin{array}{ll}
\left\{P\left(q_{i}(\theta)\right)-\theta\right\} q_{i}(\theta)-c+t(\theta) & \text { if } \phi \geqslant K(\theta) \\
\{P(Q(\theta, \phi))-\theta\} q_{i}(\theta)-c+t(\theta)+\alpha(\theta) q_{e}(\theta, \phi)+A(\theta) & \text { if } \phi<K(\theta)
\end{array} .\right.
$$

\section{First-best scenario}

Consider the first-best situation with known $\theta$ and $\phi$. Given the welfare function $W=$ $S(Q)-P(Q) Q+\beta\left\{\pi_{i}+\pi_{e}\right\}-t$, the first-best solution is

FB (1). If $\theta \leqslant \phi$ then $q_{i}=a-\theta / b, A=\alpha=0, t=c$ and hence $q_{e}=0$.

FB (2). If $\theta>\phi$ then $q_{i}=0, \alpha=-b q_{e}, A=b q_{e}^{2}, t=c$ and $q_{e}=a-\theta / b$.

The efficient market structure corresponds to a situation where either the entrant or the incumbent acts as a monopolist. The operating firm is the one with lower marginal cost. The problem with such a first-best mechanism lies in its implementability arising from the open-access nature of our framework. As can be seen, to achieve the first-bests the access charge should be contingent on the cost $\phi$, contradicting our assumption that the mechanism must be non-discriminatory. In Armstrong (2001), the regulator achieves the first-best solution in an open-access environment with an ECPR access charge. However, the firms compete $\dot{a}$ la Bertrand and the market is entirely served by the firm with lower marginal cost; therefore there is no point in regulating the supply of the firms, and the only reason for access charge is to ensure an efficient market structure - which the ECPR achieves. ${ }^{4}$ In our model, firms compete in quantities. So being more costeffective is not enough to exclude the rival from the market. Thus, the likely market structures in our case are (i) a duopoly or (ii) a monopoly incumbent.

\section{INDIRECT REGULATION}

We now consider the mechanism design problem of the regulator when the entrant's cost is not known. Consider any mechanism $M_{i r}(\cdot)$. Given that the marginal cost of the potential entrant is unknown and that the incumbent's marginal cost is known, the expected profit of the incumbent in stage one is $\Pi_{i}(\theta)=\int_{\phi=\underline{\theta}}^{\phi=\theta} \pi_{i}(\theta, \phi) g(\phi) \mathrm{d} \phi$. Simplifying the expected profit of the incumbent, we get

$$
\Pi_{i}(\theta)=\int_{\phi=\underline{\theta}}^{\phi=K(\theta)} \mu(\theta, \phi) g(\phi) \mathrm{d} \phi+\{1-G(K(\theta))\} P\left(q_{i}(\theta)\right) q_{i}(\theta)-c-\theta q_{i}(\theta)+t(\theta),
$$


where $\mu(\theta, \phi)=P(Q(\theta, \phi)) q_{i}(\theta)+\alpha(\theta) q_{e}(\theta, \phi)+A(\theta)$. Given any $\theta \in[\underline{\theta}, \bar{\theta}]$, the regulator's objective is to select $M_{i r}(\theta)=\left\langle q_{i}(\theta), A(\theta), \alpha(\theta), t(\theta)\right\rangle$ to maximize

$$
\begin{aligned}
E_{\phi}(W(\theta, \phi))= & \int_{\phi=\underline{\theta}}^{\phi=\bar{\theta}}\left[S(Q(\theta, \phi))-P(Q(\theta, \phi)) Q(\theta, \phi)+\beta\left\{\pi_{i}(\theta, \phi)+\pi_{e}(\theta, \phi)\right\}\right. \\
& -t(\theta)] g(\phi) \mathrm{d} \phi
\end{aligned}
$$

subject to the participation constraint of the incumbent (i.e. subject to $\Pi_{i}(\theta) \geqslant 0$ ). By assuming that the entrant's marginal cost follows uniform distribution, we obtain the optimal mechanism of the regulator. This optimization problem is non-concave for a certain range of $\beta$. In such a situation a characterization of the solution is possible by bunching types appropriately. The reason for non-concavity is that the expected surplus is a cubical equation in quantity of the incumbent..$^{5}$

To make the exposition of our first proposition transparent, we introduce the following function. We define

$$
\bar{H}_{\beta}(\theta)=\underline{\theta}+\left(\frac{11-6 \beta}{56-32 \beta}\right)\left[D_{\beta}(\underline{\theta})-\mathscr{2}(\theta, \beta) D_{\beta}(\theta)\right]
$$

where

$$
D_{\beta}(\theta)=\sqrt{(11-6 \beta)^{2} \Delta^{2}-2(56-32 \beta) \Delta(\theta-\underline{\theta})}
$$

and $\mathscr{Q}(\theta, \beta)=1$ if $D_{\beta}(\theta) \geqslant 0$ and $\mathscr{2}(\theta, \beta)=0$ if $D_{\beta}(\theta)$ is not a real number. One can show that $\bar{H}_{\beta}(\theta)$ is a real valued function which is non-decreasing and convex in $\theta \in[\underline{\theta}, \bar{\theta}]$. Moreover, $\bar{H}_{\beta}(\underline{\theta})=\underline{\theta}$ and $\bar{H}_{\beta}(\theta)>\theta$ for all $\theta \in[\underline{\theta}, \bar{\theta}]$. The dummy function $\mathscr{Q}(\theta, \beta)$ takes care of the non-concavity of the regulators' optimization problem by allowing for bunching whenever $D_{\beta}(\theta)$ is not a real number.

Proposition 1. Given any $\beta \in[0,1]$, the optimal indirect regulatory mechanism $M_{i r}^{f}(\theta)=$ $\left\langle q_{i}^{f}(\theta), A^{f}(\theta), \alpha^{f}(\theta), t^{f}(\theta)\right\rangle$ specifies that, $\forall \theta \in[\underline{\theta}, \bar{\theta}]$,

1. $q_{i}^{f}(\theta)=a-\bar{H}_{\beta}(\theta) / b$,

2. $A^{f}(\theta)=r_{1}(\beta) S_{\beta}^{2}(\theta) / b$,

3. $\alpha^{f}(\theta)=-r_{2}(\beta) S_{\beta}(\theta)$ and

4. $t^{f}(\theta)=c-\left[4\left(1+\beta r_{2}(\beta)\right) S_{\beta}^{3}(\theta)\right] / b \Delta$,

where $r_{1}(\beta)=6-4 \beta, r_{2}(\beta)=5-2 \beta$ and $S_{\beta}(\theta)=\left(\bar{H}_{\beta}(\theta)-\underline{\theta}\right) /\left(r_{1}(\beta)+r_{2}(\beta)\right)$.

With indirect regulation, the market price is above the incumbent's marginal cost if entry does not occur; that is, $P\left(q_{i}^{f}(\theta)\right)=\bar{H}_{\beta}(\theta)>\theta$ for all $\theta \in[\underline{\theta}, \bar{\theta}]$. This means that the incumbent produces less compared with the regulated monopoly regime. However, if entry takes place the market price falls below the incumbent's marginal cost. Interestingly, given any $\beta \in[0,1]$, the expected price is such that the incumbent realizes a zero expected profit from its downstream operations; i.e. $E_{\phi}(P(Q(\theta, \phi))=\theta$ for all $\theta$ such that $Q(\theta, \beta)=1$. Even though the expected price is identical to the price under the direct regulation monopoly outcome, under indirect regulation competition increases welfare because the financial contribution of the regulator is lower $\left(t^{f}(\theta)<c=t^{f}(\underline{\theta})\right.$ for all $\left.\theta \in(\underline{\theta}, \bar{\theta}]\right)$.

If the potential entrant decides to enter, then the entrant has to pay a lump-sum access charge to the incumbent and the entrant receives a per-unit subsidy from the incumbent. The reason for per-unit subsidy is to reduce the market power of the entrant. 
If there is no per-unit subsidy, then the entrant would have supplied an output less than the competitive output (since Stackelberg-follower output is lower than the competitive output). By setting $\alpha^{f}(\theta)<0$ for all $\theta \in(\underline{\theta}, \bar{\theta}]$, the regulator artificially reduces the entrant's marginal cost and thereby partially offsets the negative effect of the entrant's market power. This result is standard in the access pricing literature (see Laffont and Tirole 2000, Chapter 3). ${ }^{6}$

In our problem, even though the subsidy reduces the market power of the entrant, there is a positive net transfer from the entrant to the incumbent; i.e.

$$
t^{f}(\theta)-c=\frac{1}{\Delta} \int_{\phi=\underline{\theta}}^{\phi=K(\theta)}\left\{A^{f}(\theta)+\alpha^{f}(\theta) q_{e}(\theta, \phi)\right\} \mathrm{d} \phi=\frac{4\left(1+\beta r_{2}(\beta)\right) S_{\beta}^{3}(\theta)}{b \Delta}>0
$$

for all $\theta \in(\underline{\theta}, \bar{\theta}]$.

The fixed part of the access charge aims partially to finance the infrastructure and to deter entry of high marginal cost potential entrants. Entry needs to be restricted optimally because the price is above marginal cost under the optimal mechanism if the incumbent remains a monopolist. This price markup attracts inefficient entrants in the downstream market. To exclude these inefficient entrants, the regulator fixes a high $A^{f}(\theta)$.

When will entry take place? The next proposition provides a complete answer to this question.

Proposition 2. The mechanism $M_{i r}^{f}(\theta)=\left\langle q_{i}^{f}(\theta), A^{f}(\theta), \alpha^{f}(\theta), t^{f}(\theta)\right\rangle$ leads to inefficient entry. Entry takes place for all $\phi \in\left[\underline{\theta}, K^{f}(\theta)\right)$ where $\underline{\theta}<K^{f}(\theta)<\theta, \forall \theta \in(\underline{\theta}, \bar{\theta}]$ and $K^{f}(\underline{\theta})=\underline{\theta}$.

To preserve financing of the essential facility, the regulator bans those entrants with marginal cost $\phi \in\left(K^{f}(\theta), \theta\right]$. These are the entrants whose cost efficiency (relative to that of the incumbent) is not 'substantial'. By limiting entry, the regulator raises the incumbent's revenue. However, all types of incumbent firms, except the most efficient one face a positive probability of entry since $\underline{\theta}<K^{f}(\theta), \forall \theta \in(\underline{\theta}, \bar{\theta}]$. When the infrastructure cost is partially financed by public transfers, it is always efficient to allow for the entry of a more efficient competitor, provided that the efficiency advantage of the competitor is sufficiently large. ${ }^{7}$ This part of the result is in sharp contrast to the result in Caillaud and Tirole (2004), where the regulator bans entry of competitors on market with high future profitability. In De Fraja (1999), where the regulator knows $\phi$ but not $\theta$, another form of inefficient entry is observed: that is $K^{f}(\theta)>\theta$, and an entrant less efficient than the incumbent could be active on the downstream market. In the following subsection we verify that our conclusion of inefficient entry is a robust one.

\section{A general result}

What happens to the mechanism $M_{i r}^{f}(\cdot)$ if we relax the assumption that the unknown cost of the entrant follows a uniform distribution? Given our assumption that the mechanism is continuous, that $g(\phi)>0$ for all $\phi \in[\underline{\theta}, \bar{\theta}]$ and that $G(\phi)$ is continuous, two important observations can be made.

1. In any continuous mechanism, $E_{\phi}(P(Q(\theta, \phi)))=\theta$ for all $\theta \in[\underline{\theta}, \bar{\theta}]$ in the nonbunching zone (see condition (iv) in the proof of Proposition 1). This implies that, given any $\beta \in[0,1]$, we will continue to have $P\left(q^{f}(\theta)\right)>\theta$ for all $\theta$ such that $\mathscr{Q}(\theta ; \beta)=1$. Unless the incumbent is the most efficient type, there is a positive probability of entry.

(C) The London School of Economics and Political Science 2007 
2. Inefficient entry will always take place. The next proposition explains this observation.

Proposition 3. For any $g(\phi)$ with the property that $g(\phi)>0$ for all $\theta \in[\underline{\theta}, \bar{\theta}]$ and $G(\phi)$ is continuous, we cannot have an optimal continuous mechanism $M_{i r}^{f}$ for which entry is efficient.

\section{Unknown cost of the incumbent}

In this sub-section we assume that marginal cost of the incumbent is private information and that its density function satisfies assumptions (1) and (2). Hence the regulator is unaware of the marginal cost of both the incumbent and the entrant. Therefore, we have an indirect regulation problem under incomplete information. The objective of the regulator now is to select $M_{i r}^{s}=\left\langle q_{i}^{s}(\theta), A^{s}(\theta), \alpha^{s}(\theta), t^{s}(\theta)\right\rangle$ that maximizes $E_{\theta}\left(E_{\phi}(W(\theta, \phi))\right)=\int_{\theta=\theta}^{\theta=\theta} E_{\phi}(W(\theta, \phi)) f(\theta) \mathrm{d} \theta$ subject to (i) the participation constraint $\Pi_{i}(\theta) \geqslant 0, \forall \theta \in[\underline{\theta}, \bar{\theta}]$ and (ii) the incentive compatible constraint: $\Pi_{i}(\theta) \geqslant \Pi_{i}\left(\theta, \theta^{\prime}\right)$, $\forall\left(\theta, \theta^{\prime}\right) \in[\underline{\theta}, \bar{\theta}]^{2}$, where $\Pi_{i}\left(\theta, \theta^{\prime}\right)=\Pi_{i}\left(\theta^{\prime}\right)-\left(\theta-\theta^{\prime}\right) q_{i}\left(\theta^{\prime}\right)$. The optimal mechanism $M_{i r}^{s}$ satisfies constraints (i) and (ii) if and only if $\forall \theta \in[\underline{\theta}, \bar{\theta}]$, the optimal quantity $q_{i}(\theta)$ is nonincreasing in $\theta$, and $\Pi_{i}(\theta)=\int_{x=\theta}^{x=\theta} q_{i}(x) \mathrm{d} x$. As in the previous case, this optimization problem may also turn out to be non-concave for certain density functions $f(\cdot)$. For example, if $f(\cdot)$ is uniform, the optimization problem is not concave. As in the previous case, we apply optimal bunching of types. ${ }^{8}$ Before stating our next proposition, we define $z_{\beta}(\theta)=\theta+(1-\beta) L(\theta)$.

Proposition 4. Given any $\beta \in[0,1]$ and assumptions 1 and 2 , the optimal mechanism is $M_{i r}^{s}(\theta)=\left\langle q_{i}^{s}(\theta), A^{s}(\theta), \alpha^{s}(\theta), t^{s}(\theta)\right\rangle$, where, for any $\theta \in[\underline{\theta}, \bar{\theta}]$,

1. $q_{i}^{s}(\theta)= \begin{cases}q^{f}\left(z_{\beta}(\theta)\right) & \forall \theta \in[\underline{\theta}, \tilde{\theta}(\beta)] \\ q^{f}\left(z_{\beta}(\tilde{\theta}(\beta))\right) & \text { otherwise }\end{cases}$

2. $A^{s}(\theta)= \begin{cases}A^{f}\left(z_{\beta}(\theta)\right) & \forall \theta \in[\underline{\theta}, \tilde{\theta}(\beta)] \\ A^{f}\left(z_{\beta}(\tilde{\theta}(\beta))\right) & \text { otherwise }\end{cases}$

3. $\alpha^{s}(\theta)= \begin{cases}\alpha^{f}\left(z_{\beta}(\theta)\right) & \forall \theta \in[\underline{\theta}, \tilde{\theta}(\beta)] \\ \alpha^{f}\left(z_{\beta}(\tilde{\theta}(\beta))\right) & \text { otherwise }\end{cases}$

and

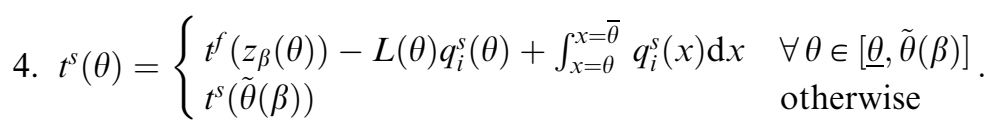

Finally, we have no pooling (that is, $\tilde{\theta}(\beta)=\bar{\theta}$ ) if $\beta$ satisfies $z_{\beta}(\bar{\theta}) \leqslant \underline{\theta}+$ $\left[\left(r_{1}(\beta)+r_{2}(\beta)\right)^{2} \Delta\right] / 16\left(r_{1}(\beta)+1\right)$, and we have partial pooling otherwise.

The indirect mechanism under asymmetric information is similar to the indirect mechanism, modulo the fact that the regulator uses the virtual marginal cost $z_{\beta}(\theta)$ for the incumbent rather than its true cost $\theta$. As in Caillaud (1990), the regulator substitutes the production of the incumbent by the production of the entrant, hence the rents paid to the incumbent are lower while the expected price remains identical $\left(E_{\phi}(P(\theta, \phi))=z_{\beta}(\theta)\right.$ for all $\left.\theta \leqslant \tilde{\theta}(\beta)\right)$. Therefore, for each $\theta \leqslant \tilde{\theta}(\beta)$, the consumer surplus

(C) The London School of Economics and Political Science 2007 
is on an average identical to the directly regulated monopoly regime under asymmetric information. Competition is welfare-enhancing because the contribution of the regulator to the infrastructure cost is lower in comparison to a monopoly solution under asymmetric information. As in the known cost case, the positive contribution of the entrant to the infrastructure cost implies a reduction of the transfer. In addition, given that the incumbent produces less when it faces the threat of entry, the information rent is also lower; hence the regulator has another reason to reduce the transfer. However, in our framework the incumbent always produces, whereas in Caillaud (1990) the incumbent is shut down when its virtual marginal cost is larger than the expected cost of the entrant. This is because the incumbent's presence enhances competition on the downstream market, which is not necessary in Caillaud's framework owing to the presence of a competitive fringe of entrants.

When concavity is not satisfied, i.e. for some $f(\theta)$ and $\theta>\tilde{\theta}(\beta)$, the optimal mechanism has a bunching zone. Bunching appears when there is a lot of entry, i.e. for the higher values of $\theta$. To overcome the existence problem and to preserve competition, the regulator sets a minimal quantity for the higher types. In the bunching region $E_{\phi}(P(\theta, \phi))=z_{\beta}(\tilde{\theta})<z_{\beta}(\theta)$ for all $\theta \in(\tilde{\theta}, \bar{\theta}]$. Consumer surplus is on an average higher than under the direct regulation monopoly regime, and competition is also welfareimproving, even if transfer may increase.

In this mechanism, entry occurs if $\phi \leqslant K^{s}(\theta)=K^{f}\left(z_{\beta}(\theta)\right)$. We now consider the question of efficient entry and compare entry levels under complete and incomplete information.

Proposition 5. Under $M_{i r}^{s}(\theta)=\left\langle q_{i}^{s}(\theta), A^{s}(\theta), \alpha^{s}(\theta), t^{s}(\theta)\right\rangle$, the following can be said about the entry limit $K^{s}(\theta)$.

1. If $\tilde{\theta}(\beta)=\bar{\theta}$, then $K^{s}(\underline{\theta})=\underline{\theta}, K^{s}(\bar{\theta})<\bar{\theta}, K^{s}(\theta)$ is strictly increasing in $\theta \in[\underline{\theta}, \bar{\theta}]$ and $K^{f}(\theta)<K^{s}(\theta)$ for all $\theta \in[\underline{\theta}, \bar{\theta}]$.

2. If $\tilde{\theta}(\beta)<\bar{\theta}$, then $K^{s}(\underline{\theta})=\underline{\theta}, K^{s}(\tilde{\theta}(\beta))<\bar{\theta}, K^{s}(\theta)$ is strictly increasing in $\theta \in(\underline{\theta}, \tilde{\theta}(\beta))$, $K^{s}(\theta)=K^{s}(\tilde{\theta}(\beta))$ for all $\theta \in[\tilde{\theta}(\beta), \bar{\theta}]$ and $K^{f}(\theta) \leqslant K^{s}(\theta)$ for all $\theta \in[\underline{\theta}, \bar{\theta}]$.

What follows from Proposition 5 is that $K^{f}(\theta)<K^{s}(\theta)$ for all $\theta \in(\underline{\theta}, \bar{\theta}(\beta))$; that is, in general, there is more entry under $M_{i r}^{s}(\theta)$ than under $M_{i r}^{f}(\theta)$. This is because the regulator allows entry by considering the gap between the incumbent's virtual marginal cost and the entrant's true marginal cost, while under complete information the regulator allows entry by considering the gap between the incumbent's true (and not virtual) marginal cost and the entrant's true marginal cost. Since the virtual marginal cost of the incumbent is larger than its true marginal cost, there is more entry under asymmetric information. The regulator allows more entry under asymmetric information simply because production of the incumbent is relatively more costly than that of the entrant. ${ }^{9}$ This result is in sharp contrast with Dana and Spier, and Caillaud and Tirole (2004), where incomplete information reduces competition. In Dana and Spier, where the market structure is regulated, monopoly production is more likely under asymmetric information. Moreover, the monopoly right is not necessarily granted to the more efficient firm. The highcost firm can operate in the market when the low-cost firm has a larger virtual marginal cost than the high-cost one.

Under $M_{i r}^{s}(\theta)$ two types of inefficient entry are possible: (A) not allowing a potential entrant, more efficient than the incumbent, to operate on the downstream market and (B) allowing a potential entrant, less efficient than the incumbent, to operate on the 


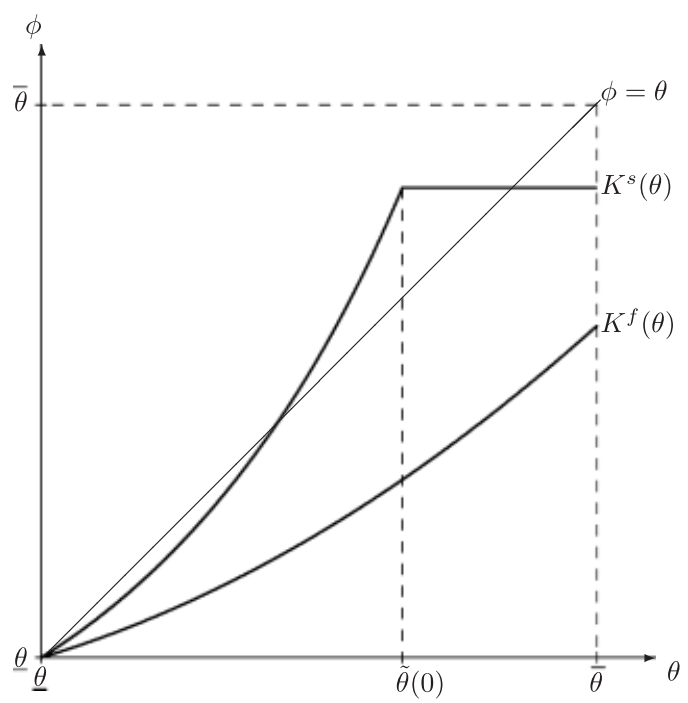

FIGURE 2. The entry levels under complete and incomplete information for $f(\theta)=g(\theta)=1 / \Delta$ and for $\beta=0$.

downstream market. The type A inefficiency follows from Proposition 5. Note that $K^{s}(\underline{\theta})=\underline{\theta}$ and $K^{s}(\bar{\theta})<\bar{\theta}\left(K^{s}(\tilde{\theta}(\beta))<\bar{\theta}\right)$ under a fully (partially) separating mechanism guarantees that there are intervals in the domain of the continuously differentiable $K^{s}(\theta)$ function such that $K^{S}(\theta)<\theta$. However, a B type inefficiency entry does not follow from Proposition 5 but depends on distribution of the marginal cost of the incumbent. It is easy to verify that if, for example, $f(\cdot)$ follows uniform distribution, that is if $f(\theta)=$ $g(\phi)=1 / \Delta$ for all $\theta \in[\underline{\theta}, \bar{\theta}]$ and if $\beta=0$, then the second type of inefficiency is also possible. With uniform distribution there will be pooling under unknown cost, and complete separation under known cost, and in particular the cut-off points are $\tilde{\theta}(0)=$ $(121 / 224) \bar{\theta}+(103 / 224) \underline{\theta}$ for the unknown cost case and $\bar{\theta}(0)=\bar{\theta}$ for the known cost case. Using $K^{s}(\tilde{\theta}(0))=(11 / 14) \bar{\theta}+(3 / 14) \underline{\theta}$, we get $K^{s}(\tilde{\theta}(0))-\tilde{\theta}(0)=(55 / 224) \Delta>0$. Hence, if the marginal cost of the incumbent follows uniform distribution, then $K^{s}(\tilde{\theta}(0))>\tilde{\theta}(0)$, which implies that there are stretches of the $K^{s}(\theta)$ function where the second type of inefficiency arises, i.e. non-empty intervals in $[\underline{\theta}, \bar{\theta}]$ where $K^{s}(\theta)>\theta$. The entry situation with $\beta=0$ and with uniform $f$ and $g$ functions is illustrated in Figure 2.

\section{SUMmARY AND CONCLUSIONS}

In this paper we provide a framework for analysing the impact of a regulatory mechanism on the market structure in the presence of incomplete information. In particular, we analyse a model of regulation in a market where a firm owns an essential facility and the market structure is endogenous. There are two players in the market: the incumbent firm (that owns the essential facility) and a potential entrant. The regulator uses four instruments to regulate this market in order to maximize social welfare. The social welfare function in our problem is general, in the sense that it allows for different welfare weights to producer's surplus. The instruments are the quantity to be produced by the incumbent, the amount of public funding to be transferred by the regulator to the incumbent, and a two part tariff to be paid by the entrant to the incumbent if the entrant 
decides to enter the market. Depending on the weight $\beta$, we can have either a completely separating solution or a partial separating solution. Our optimal indirect regulatory mechanisms takes care of the conflict between efficient market structure and infrastructure financing that results from incomplete information and the provision of non-discriminatory access of the essential facility to the entrant.

The main conclusion of this paper is that granting non-discriminatory access of the essential facility to a competitor is welfare-improving compared with the monopoly outcome, though such non-discriminatory access leads to inefficient entry.

With, a known marginal cost of the incumbent, we achieve a welfare improvement with the optimal indirect regulation mechanism relative to the regulated monopoly regime. This welfare improvement is mainly due to the contribution of the entrant to infrastructure financing. Competition leads to a larger consumer surplus only when there is bunching of less efficient incumbents (a pooling situation). In all the other cases the expected price is the same as in the regulated monopoly regime, and competition allows the regulator to reduce her transfer. As a result, we have a rise in social welfare. An entry ban of more efficient competitors aims to finance the infrastructure. By allowing only the entry of a competitor with a sufficiently large cost advantage, the regulator can extract a larger contribution towards the cost of the essential input.

With the marginal cost of the incumbent unknown, we also achieve welfare improvement with the optimal indirect regulation mechanism in comparison with the regulated monopoly regime. This welfare rise is not only because of the contribution of the entrant to infrastructure financing, but also because of the lower information rent paid to the incumbent, which is achieved by substituting incumbent's output with the entrant's output. Hence in this case we have more entry compared with our indirect regulation mechanism where the marginal cost of the incumbent is known. Depending on the distribution of the incumbent's marginal cost, the other form of inefficiency arises, i.e. that of a less efficient competitor entering the market.

In a monopoly regulation problem, if the cost of the monopolist is unknown and the regulator has limited funds, the optimal mechanism is a third-best one, since optimal output with limited funds is lower than the second-best output (see Gautier and Mitra 2006). Allowing for endogenous market structure and indirect regulation can help in this sort of a limited fund situation in two ways. First, with indirect regulation we have a lower transfer of public funding (from the regulator to the incumbent) relative to both second-best and third-best regulated monopoly situation. Second, owing to potential competition in the downstream market, the expected output in the market is also higher relative to the second-best and hence third-best monopoly output.

The two-part tariff plays an important and indirect role in regulating the entrant. Recall that this two-part tariff is contingent on the type of the incumbent. The fixed part of the two-part tariff helps to deter entrants lacking adequate cost advantage relative to the incumbent. Once the entrant is efficient enough and decides to enter the market, we have a Stackelberg leader-follower situation which generates output below the competitive level. In order to reduce this output inefficiency, the entrant gets a perunit subsidy from the incumbent so that this output gap is reduced.

In the open-access environment with non-discriminatory access charges, the market structure is necessarily inefficient under optimal regulation. It may seem that one possible way out of this problem would be to specify two production levels for the incumbent, where one level applies if entry does not occur and the other level applies if entry does occur. However, even then we cannot have efficient market structure simply because it is not possible to regulate the production of the entrant. Hence maintaining a positive

(C) The London School of Economics and Political Science 2007 
production level for the incumbent, even though the entrant is more efficient, is a means of disciplining the entrant. Moreover, it is also not optimal to ensure efficient entry simply because the regulator continues to face the same trade-off between restricting entry and increasing the financial contribution of the firms active on the market. Therefore, it is an open question whether more sophisticated regulatory instruments such as more general tariffs can solve the twin problem of inefficient market structure and inefficient entry.

Following Caillaud (1990), we considered a scenario where the regulator specifies the regulatory environment before the competitor decides to enter the downstream market. It is an open question as to what will happen if we reverse the sequence of moves, that is if we consider a regulatory environment in which, the first stage, the entrant announces its quantity and, contingent on this announcement, in the second stage the regulator specifies (i) the quantity to be produced by the incumbent, (ii) access charges to be paid by the entrant to the incumbent and (iii) the amount of public funding that the incumbent will receive from the regulator. Clearly, that analysis will depend on the regulator's second-stage knowledge of the entrant's expected profit conditional on the entrant's first-stage announced quantity. However, unless there is some interdependence between the (announced) quantity of the entrant and its (unknown) marginal cost which is known to the regulator, there will be inefficient entry. Hence we feel that changing the sequence of moves cannot achieve much when cost of the entrant is unknown. A regulatory mechanism contingent on output of the entrant can be very effective if, instead of changing the sequence of moves, we consider a model of repeated interaction where the objective is to achieve 'dynamic' market efficiency. In such a scenario the regulatory contract of the incumbent from the second period onwards will be contingent on the entry decisions (and hence output decisions) of the entrant. This is clearly a very good open question.

\section{APPENDIX}

\section{Proof of Proposition 1}

Given the optimal entry decision of stage 2, in stage 1 the regulator maximizes $E_{\phi}(W(\theta, \phi))=$ $\int_{\phi=\theta}^{\phi=\bar{\theta}} W(\theta, \phi) g(\phi) \mathrm{d} \phi$ where $W(\theta, \phi)=\frac{b}{2} Q^{2}(\theta, \phi)+\beta\left\{\pi_{i}(\theta, \phi)+\pi_{e}(\phi, \theta)\right\}-t(\theta)$. Using $\bar{\pi}_{e}(\theta, \phi)=$ $b q_{e}^{2}(\phi)-A(\theta)$ and $E_{\phi}\left(\pi_{i}(\theta, \phi)\right)=0$ we get

$$
\begin{aligned}
E_{\phi}(W(\theta, \phi))= & (a-\theta) q_{i}(\theta)-\frac{b q_{i}^{2}(\theta)}{2} \\
& +\int_{\phi=\underline{\theta}}^{\phi=K(\theta)}\left\{\left(\frac{1}{2}+\beta\right) b q_{e}^{2}(\phi)+\alpha(\theta) q_{e}(\phi)+(1-\beta) A(\theta)\right\} g(\phi) \mathrm{d} \phi-c .
\end{aligned}
$$

Simplification $E_{\phi}(W(\theta, \phi))$ we get

(A) $\quad E_{\phi}(W(\theta, \phi))=(a-\theta) q_{i}(\theta)-\frac{b q_{i}^{2}(\theta)}{2}+y(\theta)\left(\frac{3 y(\theta)+2 \alpha(\theta)}{2 b}\right) G(K(\theta))+P_{\beta}(\theta)-c$ where $y(\theta)=\sqrt{b A(\theta)}$,

$$
P_{\beta}(\theta)=\left(\frac{1+2 \beta}{8 b}\right) I_{2}(K(\theta))+\left(\frac{(1+2 \beta) y(\theta)+\alpha(\theta)}{2 b}\right) I_{1}(K(\theta))
$$


and $I_{i}(K(\theta))=\int_{\phi=\theta}^{\phi=K(\theta)}(K(\theta)-\phi)^{i} g(\phi) \mathrm{d} \phi$ for $i \in\{1,2\}$. Integrating by parts we get $I_{1}(K(\theta))=$ $\int_{\phi=\underline{\theta}}^{\phi=K(\theta)} G(\phi) \mathrm{d} \phi$ and $I_{2}(K(\theta))=2 \int_{\phi=\underline{\theta}}^{\phi=K(\theta)} \mathbf{G}(\phi) \mathrm{d} \phi$ where $\mathbf{G}(\phi)=\int_{x=\underline{\theta}}^{x=\phi} G(x) \mathrm{d} x$. The first-order conditions with respect to $q_{i}(\theta), \alpha(\theta)$ and $A(\theta)$ gives

(i) $\left[P\left(q_{i}(\theta)\right)-\theta\right]-y(\theta)\left(\frac{3 y(\theta)+2 \alpha(\theta)}{2}\right) g(K(\theta))=b \frac{\partial P_{\beta}(\theta)}{\partial K(\theta)}$,

(ii) $\frac{I_{1}(K(\theta))}{2 b}+\frac{y(\theta) G(K(\theta))}{b}-y(\theta)\left(\frac{3 y(\theta)+2 \alpha(\theta)}{2 b}\right) g(K(\theta))=\frac{\partial P_{\beta}(\theta)}{\partial K(\theta)}$ and

(iii) $\frac{(1+2 \beta) I_{1}(K(\theta))}{4}+\frac{[3 y(\theta)+\alpha(\theta)] G(K(\theta))}{2}-\frac{[y(\theta)(3 y(\theta)+2 \alpha(\theta)) g(K(\theta))]}{2}=b \frac{\partial P_{\beta}(\theta)}{\partial K(\theta)}$.

In the above conditions,

$$
\frac{\partial P_{\beta}(\theta)}{\partial K(\theta)}=\left(\frac{1+2 \beta}{4 b}\right) \mathbf{G}(K(\theta))+\left(\frac{(1+2 \beta) y(\theta)+\alpha(\theta)}{2 b}\right) G(K(\theta)) .
$$

Simplifying (ii) we get

$$
\int_{\phi=\underline{\theta}}^{\phi=K(\theta)} q_{e}(\phi) g(\phi) \mathrm{d} \phi-y(\theta)\left(\frac{3 y(\theta)+2 \alpha(\theta)}{2 b}\right) g(K(\theta))=\frac{\partial P_{\beta}(\theta)}{\partial K(\theta)}
$$

From conditions (i) and (ii*) it follows that expected market price equals the marginal cost of the incumbent; that is

(iv) $E_{\phi}(P(Q(\theta, \phi)))=\theta \forall \theta \in[\underline{\theta}, \bar{\theta}]$.

Using $I_{1}(K(\theta))=\mathbf{G}(K(\theta))$ in (ii) and (iii), we get

(v) $\left(\frac{1-2 \beta}{2}\right) \mathbf{G}(K(\theta))=[y(\theta)+\alpha(\theta)] G(K(\theta))$.

Finally, from (i) and (v), we get

$$
\begin{aligned}
P\left(q_{i}(\theta)\right)-\theta= & y(\theta)\left(\frac{3 y(\theta)+2 \alpha(\theta)}{2}\right) g(K(\theta))+\left(\frac{1+2 \beta}{4}\right) \mathbf{G}(K(\theta)) \\
& +\left(\frac{(1+2 \beta) y(\theta)+\alpha(\theta)}{2}\right) G(K(\theta)) .
\end{aligned}
$$

Assuming uniform distribution of $\phi$, we get two possible solutions. One is

$$
\begin{aligned}
\text { [1] } q_{i}^{f}(\theta) & =\frac{a-H_{\beta}(\theta)}{b}, \alpha^{f}(\theta)=-\left(\frac{5-2 \beta}{11-6 \beta}\right)\left(H_{\beta}(\theta)-\underline{\theta}\right) \text { and } \\
A^{f}(\theta) & =\left(\frac{6-4 \beta}{11-6 \beta}\right)^{2} \frac{\left(H_{\beta}(\theta)-\underline{\theta}\right)^{2}}{b} .
\end{aligned}
$$

Here

$$
\begin{aligned}
& H_{\beta}(\theta)=\underline{\theta}+\left(\frac{11-6 \beta}{56-32 \beta}\right)\left[D_{\beta}(\underline{\theta})-D_{\beta}(\theta)\right] \text { and } \\
& D_{\beta}(\theta)=\sqrt{(11-6 \beta)^{2} \Delta^{2}-2(56-32 \beta) \Delta(\theta-\underline{\theta})} .
\end{aligned}
$$

Substituting $r_{1}(\beta)=6-4 \beta, r_{2}(\beta)=5-2 \beta$ and $S_{\beta}(\theta)=\left(H_{\beta}(\theta)-\underline{\theta}\right) /\left(r_{1}(\beta)+r_{2}(\beta)\right)$ we get $q^{f}(\theta)$, $\alpha^{f}(\theta)$ and $A^{f}(\theta)$ of this Proposition provided $2(\theta ; \beta)=1$. The other possible solution is the one where we have complete entry ban; that is, $\forall \theta$,

(C) The London School of Economics and Political Science 2007 


$$
\text { [2] } q_{i}^{f}(\theta)=\frac{a-\theta}{b}, \alpha^{f}(\theta)=-3(\theta-\underline{\theta}) \quad \text { and } \quad A^{f}(\theta)=\frac{4}{b}(\theta-\underline{\theta})^{2} .
$$

Complete separation of solution [1] exists only if $\beta$ is such that $\theta \in[\underline{\theta}, \bar{\theta}]$ such that $\nexists D_{\beta}(\theta)$ is a complex number (with non-zero imaginary part). It is quite easy to check that $D_{\beta}(\theta)$ satisfies this restriction if and only if $\beta \in[0, \hat{\beta}]$, where $\hat{\beta}=(17-4 \sqrt{3}) / 18$. Hence, for $\beta \in[0, \hat{\beta}]$, both solutions are feasible. However the solution that leads to a higher welfare will be optimum. Hence to prove the proposition it is necessary to show that solution [1] leads to a higher welfare than solution [2]. Let $W_{[\mathrm{i}]}(\theta)$ denote the expected welfare with solution [i] for $\mathrm{i} \in\{1,2\}$ and $\mathbf{D i}(\theta)=W_{[1]}(\theta)-W_{[2]}(\theta)$. Using the two solutions, we get

$$
\mathbf{D i}(\theta)=\frac{(1-\beta)^{2}\left(H_{\beta}(\theta)-\theta\right)\left(H_{\beta}(\theta)-\underline{\theta}\right)}{3 b \gamma(\beta)}\left\{1-\frac{3}{4}\left[\frac{\gamma(\beta)\left[D_{\beta}(\underline{\theta})-D_{\beta}(\theta)\right]}{(1-\beta)^{2}(56-32 \beta) \Delta}\right]\right\},
$$

where $\gamma(\beta)=\left(77-86 \beta+24 \beta^{2}\right) /\left(77-16 \beta-28 \beta^{2}\right)$. In (A1), (a) $0<\gamma(\beta) \leqslant 1$ for all $\beta \in[0,1]$, (b) $0<\left[D_{\beta}(\underline{\theta}) /(1-\beta)^{2}(56-34 \beta)\right]<\frac{4}{3}$ for all $\beta \in[0,1]$ and (c) $D_{\beta}(\underline{\theta})>D_{\beta}(\theta)$ for all $\theta \in(\underline{\theta}, \bar{\theta}]$. Using (a), (b) and (c) in (A1), we get $\mathbf{D i}(\theta)>0$ for all $\theta \in(\underline{\theta}, \bar{\theta}]$ and $\mathbf{D i}(\underline{\theta})=0$. Therefore we have established that, except for $\underline{\theta}$, solution [1] leads to a strictly higher welfare than solution [2].

To check the second-order condition with solution [1], we first incorporate the optimal values of $\alpha^{f}(\theta)=-[(5-2 \beta) /(11-6 \beta)]\left(P\left(q_{i}(\theta)\right)-\underline{\theta}\right)$ and $A^{f}(\theta)=\frac{1}{b}[(6-4 \beta) /(11-6 \beta)]^{2}\left(P\left(q_{i}(\theta)\right)-\underline{\theta}\right)^{2}$ as a function of the quantity $q_{i}(\theta)$ in $W_{[1]}(\theta)$. This gives

$$
W_{[1]}(\theta)=(a-\theta) q_{i}(\theta)-\frac{b\left\{q_{i}(\theta)\right\}^{2}}{2}+\frac{4(1-\beta)^{2}\left(77-16 \beta-28 \beta^{2}\right)\left(P\left(q_{i}(\theta)\right)-\underline{\theta}\right)^{3}}{3 b \Delta(11-6 \beta)^{3}}-c .
$$

The second derivative of $W_{[1]}(\theta)$ with respect to $q_{i}(\theta)$ gives

$$
\frac{\partial^{2} W_{[1]}(\theta)}{\partial q_{i}(\theta)^{2}}=-b\left\{1-\left[\frac{8(1-\beta)^{2}\left(77-16 \beta-28 \beta^{2}\right)\left[D_{\beta}(\theta)-D_{\beta}(\theta)\right]}{(11-6 \beta)^{3}(56-32 \beta) \Delta}\right]\right\} .
$$

Clearly, $\partial^{2} W_{[1]}(\theta) / \partial q_{i}(\theta)^{2}<0 \forall \theta \in[\underline{\theta}, \bar{\theta}]$. Hence the optimal solution is [1]. From the value of $H_{\beta}(\theta)$, it is immediate that $H_{\beta}(\underline{\theta})=\underline{\theta}$. Using some simple calculations, we can show that $H_{\beta}(\theta)>\theta$ for all $\theta \in(\underline{\theta}, \bar{\theta}]$. By differentiating $H_{\beta}(\bar{\theta})$ twice with respect to $\theta$, we get that $H_{\beta}(\theta)$ is increasing and strictly convex in $\theta \in(\underline{\theta}, \bar{\theta})$. Moreover, with solution [1] there is entry possibility and the entry limit is $K(\theta)=\underline{\theta}+$ $[(1-\beta) /(14-8 \beta)]\left[D_{\beta}(\underline{\theta})-D_{\beta}(\theta)\right]$. The optimal transfer is obtained from $\Pi_{i}(\theta)=0$, which implies that

$$
\frac{1}{\Delta}\left[\int_{\phi=\underline{\theta}}^{\phi=K^{f}(\theta)} \mu^{f}(\theta ; \phi) \mathrm{d} \phi+\int_{\phi=K^{f}(\theta)}^{\phi=\bar{\theta}} P\left(q_{i}^{f}(\theta)\right) q_{i}^{f}(\theta) \mathrm{d} \phi\right]-c-\theta q_{i}^{f}(\theta)+t^{f}(\theta)=0,
$$

where $\mu^{f}(\theta ; \phi)=P\left(Q^{f}(\theta, \phi)\right) q_{i}^{f}(\theta)+\alpha^{f}(\theta) q_{e}(\theta, \phi)+A^{f}(\theta)$. At the optimum, the expected market price equals the type of the incumbent. Therefore,

$$
E_{\phi}\left(P\left(Q^{f}(\theta, \phi)\right)\right)=\frac{1}{\Delta}\left[\int_{\phi=\underline{\theta}}^{\phi=K^{f}(\theta)} P\left(Q^{f}(\theta, \phi)\right) \mathrm{d} \phi+\int_{\phi=K^{f}(\theta)}^{\phi=\bar{\theta}} P\left(q_{i}^{f}(\theta)\right) \mathrm{d} \phi\right]=\theta
$$

Substituting (A5) in (A4) and simplifying, we get

$$
t^{f}(\theta)=c-\frac{1}{\Delta}\left[\int_{\phi=\underline{\theta}}^{\phi=K^{f}(\theta)}\left\{\alpha^{f}(\theta) q_{e}(\theta, \phi)+A^{f}(\theta)\right\} \mathrm{d} \phi\right]
$$

for all $\theta \in[\underline{\theta}, \bar{\theta}]$. Observe that the expected revenue of the incumbent is strictly positive; that is,

$$
\begin{aligned}
\frac{1}{\Delta}\left[\int_{\phi=\underline{\theta}}^{\phi=K^{f}(\theta)}\left\{\alpha^{f}(\theta) q_{e}(\theta, \phi)+A^{f}(\theta)\right\} \mathrm{d} \phi\right] & =\frac{4\left(1+5 \beta-2 \beta^{2}\right)\left(H_{\beta}(\theta)-\underline{\theta}\right)^{3}}{(11-6 \beta)^{3} b \Delta} \\
& =\frac{4\left(1+\beta r_{2}(\beta)\right)}{b \Delta}\left\{S_{\beta}(\theta)\right\}^{3}>0
\end{aligned}
$$

(C) The London School of Economics and Political Science 2007 
for all $\theta \in(\underline{\theta}, \bar{\theta}]$, which implies that $t^{f}(\theta)<c$ for all $\theta \in(\underline{\theta}, \bar{\theta}]$. Using $H_{\beta}(\underline{\theta})=\underline{\theta}$ we get $t^{f}(\underline{\theta})=c$. Thus, we have established the solution for $\beta \in[0, \hat{\beta}]$.

For $\beta \in[\hat{\beta}, 1]$ we have a concavity problem. To solve this non-existence problem with pointwise optimization, we incorporate an optimum bunching procedure. It is clear that the optimal cut-off point is $\bar{\theta}(\beta)$, where $\bar{\theta}(\beta)$ has the property that, for all $\theta \in[\bar{\theta}(\beta), \bar{\theta}], \mathscr{2}(\theta ; \beta)=0$. Therefore, $\bar{\theta}(\beta)$ is the point from which we have an existence problem with pointwise maximization. Thus, the optimal solution is identical to the pointwise optimization problem in the well defined zone and is a pooling one for higher types. Finally, we verify that $\mathbf{D i}(\theta) \geqslant 0$ for all $\theta$. It is obvious that $\mathbf{D i}(\theta) \geqslant 0 \forall$ $\theta \in[\underline{\theta}, \bar{\theta}(\beta)]$. For $\theta \in(\bar{\theta}(\beta), \bar{\theta}]$ we get

$$
\mathbf{D i}(\theta)=\mathbf{D i}(\bar{\theta}(\beta))+\left(\frac{\theta-\bar{\theta}(\beta)}{2 b}\right)\left(H_{\beta}(\bar{\theta}(\beta))+\bar{\theta}(\beta)-2 \theta\right) .
$$

Using $\quad H_{\beta}(\bar{\theta}(\beta))=\underline{\theta}+\left[(11-6 \beta)^{2} \Delta /(56-32 \beta)\right], \quad \bar{\theta}(\beta)=\underline{\theta}+\left[(11-6 \beta)^{2} \Delta / 2(56-32 \beta)\right] \quad$ and $H_{\beta}(\bar{\theta}(\beta))+\bar{\theta}(\beta)-2 \theta \geqslant H_{\beta}(\bar{\theta}(\beta))+\bar{\theta}(\beta)-2 \bar{\theta}$, we see that the right-hand side of (10) is not smaller than $M(\beta)=\operatorname{Di}(\bar{\theta}(\beta))+[(\theta-\bar{\theta}(\beta)) \Delta / 4 b(56-32 \beta)]\left[3(11-6 \beta)^{2}-4(56-32 \beta)\right]$. The number $M(\beta)>0$ for all $\beta \in[\hat{\beta}, 1]$. Hence the welfare under the partial bunching case dominates the monopoly outcome.

To capture the monopoly price under both the no-pooling and partial-pooling cases, we define $\bar{H}_{\beta}(\theta)=\underline{\theta}+[(11-6 \beta) /(56-32 \beta)]\left[D_{\beta}(\underline{\theta})-\mathscr{2}(\theta ; \beta) D_{\beta}(\theta)\right]$ where $\mathscr{2}(\theta ; \beta) \in\{0,1\}$ takes care of the non-concavity problem that can arise for $\beta>(17-4 \sqrt{3}) / 18$. Note that if $\mathscr{Q}(\theta ; \beta)=1$ then $\bar{H}_{\beta}(\theta)=$ $H_{\beta}(\theta)$ and if $\mathscr{Q}(\theta ; \beta)=0$ then $\bar{H}_{\beta}(\theta)=\underline{\theta}+[(11-6 \beta) /(56-32 \beta)] D_{\beta}(\underline{\theta})$. Finally, we show that $(a)$ if $2(\theta ; \beta)=1$ then $\bar{H}_{\beta}(\theta)=H_{\beta}(\theta)$ and $H_{\beta}(\theta)$ is increasing and strictly convex, and $(b)$ given any $\beta>\hat{\beta}, \bar{H}_{\beta}(\theta)$ is horizontal to the $\theta$ plane for all $\theta \in[\bar{\theta}(\beta), \bar{\theta}]$. From $(a)$ and $(b)$ it follows that, for any given $\beta \in[0,1], \bar{H}_{\beta}(\theta)$ is non-decreasing and convex.

\section{Proof of Proposition 2}

If $\beta \in[0, \hat{\beta}]$ then, $\mathscr{Q}(\theta ; \beta)=1$ and $\bar{H}_{\beta}(\theta)=H_{\beta}(\theta)$ and $M_{i r}^{f}(\theta)$ is such that

$$
K^{f}(\theta)=\left(\frac{r_{2}(\beta)-1}{r_{1}(\beta)+r_{2}(\beta)}\right) H_{\beta}(\theta)+\left(\frac{r_{1}(\beta)+1}{r_{1}(\beta)+r_{2}(\beta)}\right) \underline{\theta} .
$$

Using $H_{\beta}(\theta)$, we get

$$
\begin{aligned}
K^{f}(\theta)= & \underline{\theta}+\left(\frac{(1-\beta)\left(r_{1}(\beta)+r_{2}(\beta)\right) \Delta}{2\left(r_{1}(\beta)+1\right)}\right) \\
& -\left(\frac{1-\beta}{2\left(r_{1}(\beta)+1\right)}\right) \sqrt{\left(r_{1}(\beta)+r_{2}(\beta)\right)^{2} \Delta^{2}-16\left(r_{1}(\beta)+1\right) \Delta(\theta-\underline{\theta})} .
\end{aligned}
$$

Here $K^{f}(\underline{\theta})=\underline{\theta}$ and

$$
K^{f}(\bar{\theta})=\underline{\theta}+\left(\frac{(1-\beta)\left\{\left(r_{1}(\beta)+r_{2}(\beta)\right)-\sqrt{4+r_{2}(\beta)-11 \beta\left(r_{1}(\beta)+r_{2}(\beta)\right)}\right\}}{2\left(r_{1}(\beta)+1\right)}\right) \Delta<\bar{\theta}
$$

since $r_{1}(\beta)+r_{2}(\beta)=11-6 \beta<14-8 \beta=2\left(r_{1}(\beta)+1\right)$. To show that $K^{f}(\theta)<\theta$ for all $\theta \in(\underline{\theta}, \bar{\theta})$, we consider $\theta-K^{f}(\theta)$. We apply proof by contradiction to show that $\theta-K^{f}(\theta)>0$. Finally, $\underline{\theta}<K^{f}(\theta)$ $\forall \theta \in(\underline{\theta}, \bar{\theta})$ follows from $K^{f}(\underline{\theta})=\underline{\theta}$ and from $\partial\left[K^{f}(\theta)\right] / \partial \theta>0$ over the relevant range.

Now consider the case where $\beta \in(\hat{\beta}, 1]$, that is where we have partial bunching with $\bar{\theta}(\beta)$ as the cut-off point. As long as $\theta \in[\underline{\theta}, \bar{\theta}(\beta)]$, the earlier arguments apply. Therefore, $K^{f}(\underline{\theta})=\underline{\theta}$ and $K^{f}(\theta)<$ $\theta$ for all $\theta \in(\underline{\theta}, \bar{\theta}(\beta)]$. Moreover, $K^{f}(\theta)=K^{f}(\bar{\theta}(\beta))<\theta$ for all $\theta \in(\bar{\theta}(\beta), \bar{\theta}]$ since $K^{\bar{f}}(\bar{\theta}(\beta))<\bar{\theta}(\beta)$. Hence, the result follows for all $\beta \in[0,1]$.

\section{Proof of Proposition 3}

Entry is efficient if the solution to problem [A], given by the first-order conditions (i), (ii) and (iii), satisfies $K(\theta)=P\left(q_{i}(\theta)\right)-\alpha(\theta)-2 y(\theta)=\theta$ (see the proof of Proposition 1). Suppose that this is 
possible for some distribution of $\phi$. We can then substitute $K(\theta)$ by $\theta$ in the first-order conditions, and this would imply that $P\left(q_{i}(\theta)\right)-\theta$ can be replaced by $\alpha+2 y(\theta)$. Therefore, the first-order conditions are:

(i) (a) $[\alpha(\theta)+2 y(\theta)]-y(\theta)\left(\frac{3 y(\theta)+2 \alpha(\theta)}{2}\right) g(\theta)=b \frac{\partial P_{\beta}(\theta)}{\partial K(\theta)}$,

(ii)(a) $\frac{I_{1}(\theta)}{2 b}+\frac{y(\theta) G(\theta)}{b}-y(\theta)\left(\frac{3 y(\theta)+2 \alpha(\theta)}{2 b}\right) g(\theta)=\frac{\partial P_{\beta}(\theta)}{\partial K(\theta)}$

(iii)(a) $\frac{(1+2 \beta) I_{1}(\theta)}{4}+\frac{[3 y(\theta)+\alpha(\theta)] G(\theta)}{2}-y(\theta)\left(\frac{3 y(\theta)+2 \alpha(\theta)}{2}\right) g(\theta)=b \frac{\partial P_{\beta}(\theta)}{\partial K(\theta)}$.

Here

$$
\frac{\partial P_{\beta}(\theta)}{\partial K(\theta)}=\left(\frac{1+2 \beta}{4 b}\right) \mathbf{G}(\theta)+\left(\frac{(1+2 \beta) y(\theta)+\alpha(\theta)}{2 b}\right) G(\theta) .
$$

Solving (i)(a) and (iii)(a) for $\alpha(\theta)$ and $y(\theta)$, we have two possible solutions:

$$
\begin{aligned}
\text { (I) } \alpha(\theta) & =\frac{(1+2 \beta) \mathbf{G}(\theta)}{2(G(\theta)-2)} \text { and } y(\underline{\theta})=0 \\
\text { (II) } \alpha(\theta) & =\frac{(\beta-1) G(\theta)(16-12 G(\theta))+3 g(\theta) \mathbf{G}(\theta)(2 \beta+1)}{2 g(\theta)(3 G(\theta)-2)} \text { and } \\
y(\theta) & =\frac{(\beta-1) G(\theta)(2 G(\theta)-4)-g(\theta) \mathbf{G}(\theta)(2 \beta+1)}{g(\theta)(3 G(\theta)-2)} .
\end{aligned}
$$

From (I) we get $P\left(q_{i}(\theta)\right)=\theta+[(1+2 \beta) \mathbf{G}(\theta)] /[2(G(\theta)-2)]$. Note that this solution corresponds to the efficient component pricing rule (ECPR). ${ }^{10}$ Using this solution in (ii)(a), efficient entry implies that, $\forall \theta \in[\underline{\theta}, \bar{\theta}]$,

$$
\frac{\mathbf{G}(\theta)(G(\theta)-1+2 \beta)}{2 b(G(\theta)-2)}=0 .
$$

Observe that for $\theta=\underline{\theta}$, (A8) is satisfied, since $\mathbf{G}(\underline{\theta})=0$. However, for all $\theta \in(\underline{\theta}, \bar{\theta}], \mathbf{G}(\underline{\theta})>0$ and hence for (A8) to hold, it is necessary that $G(\theta)=1-2 \beta$, which is a constant (for any given $\beta \in[0,1]) \forall \theta \in(\underline{\theta}, \bar{\theta}]$. This is impossible given $g(\phi)>0$ for all $\phi \in(\underline{\theta}, \bar{\theta}]$. Hence ECPR is not a solution to problem $[\mathbf{A}]$.

For solution (II), we substitute the values of $\alpha(\theta)$ and $y(\theta)$ in (ii)(a). After simplifications, we see that efficient entry implies, $\forall \theta \in[\underline{\theta}, \bar{\theta}]$,

$$
\frac{4 G(\theta)^{2}(\beta-1)(G(\theta)-1)+g(\theta) \mathbf{G}(\theta)(G(\theta)(1-4 \beta)+(2 \beta-1))}{2 b g(\theta)(3 G(\theta)-2)}=0 .
$$

Note first that, for $\theta=\underline{\theta},(12)$ is satisfied since $\mathbf{G}(\underline{\theta})=G(\underline{\theta})=0$. Consider any $\theta \in(\underline{\theta}, \bar{\theta}]$. If $\exists \theta \in(\underline{\theta}, \bar{\theta}]$ such that $G(\theta)=\frac{2}{3}$, then the solution does not exist, implying impossibility. If, however, $G(\theta) \neq \frac{2}{3}$ for all $\theta \in(\underline{\theta}, \bar{\theta}]$, there is a discontinuous jump in the $G(\cdot)$ function in the interval $(\underline{\theta}, \bar{\theta}]$, which is not possible since we have assumed that $G(\cdot)$ is a continuous function and the mechanism is continuous. Hence in both cases solution (II) is not possible and we have a contradiction.

\section{Proof of Proposition 4}

The regulator's objective is to select the regulatory mechanism $M_{i r}^{s}=\left\langle q_{i}^{s}(\theta), A^{s}(\theta), \alpha^{s}(\theta), t^{s}(\theta)\right\rangle$ that maximizes $\int_{\theta=\underline{\theta}}^{\theta=\bar{\theta}} E_{\phi}(W(\theta, \phi)) f(\theta) \mathrm{d} \theta \quad$ where $\quad W(\theta, \phi)=\frac{b}{2} Q^{2}(\theta, \phi)+\beta\left\{\pi_{i}(\theta, \phi)+\pi_{e}(\phi, \theta)\right\}-t(\theta)$ and $E_{\phi}(W(\theta, \bar{\phi}))=\int_{\phi=\underline{\theta}}^{\phi=\bar{\theta}} W(\theta, \phi) g(\phi) \mathrm{d} \phi$. The constraints faced by the regulator are (a)

(C) The London School of Economics and Political Science 2007 
$\Pi_{i}(\theta) \geqslant \Pi_{i}\left(\theta ; \theta^{\prime}\right), \forall\left(\theta, \theta^{\prime}\right) \in[\underline{\theta}, \bar{\theta}]^{2}$ and (b) $\Pi_{i}(\theta) \geqslant 0, \forall \theta \in[\underline{\theta}, \bar{\theta}]$. Using $\pi_{e}(\theta, \phi)=b q_{e}^{2}(\phi)-A(\theta)$ in the surplus term we get

$$
\begin{aligned}
E_{\phi}(W(\theta, \phi))= & (a-\theta) q_{i}(\theta)-\frac{b q_{i}^{2}(\theta)}{2} \\
& +\int_{\phi=\underline{\theta}}^{\phi=K(\theta)}\left\{\left(\frac{1}{2}+\beta\right) b q_{e}^{2}(\phi)+\alpha(\theta) q_{e}(\phi)+(1-\beta) A(\theta)\right\} g(\phi) \mathrm{d} \phi \\
& -(1-\beta) \Pi_{i}(\theta)-c .
\end{aligned}
$$

After simplification, we get

$$
\begin{aligned}
{[\overline{\mathbf{A}}] \quad E_{\phi}(W(\theta, \phi))=} & (a-\theta) q_{i}(\theta)-\frac{b q_{i}^{2}(\theta)}{2}+y(\theta)\left(\frac{3 y(\theta)+2 \alpha(\theta)}{2 b}\right) G(K(\theta))+P_{\beta}(\theta) \\
& -(1-\beta) \Pi_{i}(\theta)-c,
\end{aligned}
$$

where

$$
\begin{aligned}
y(\theta) & =\sqrt{b A(\theta)}, P_{\beta}(\theta) \\
& =\left(\frac{1+2 \beta}{4 b}\right) \int_{\phi=\underline{\theta}}^{\phi=K(\theta)} \mathbf{G}(\phi) \mathrm{d} \phi+\left(\frac{(1+2 \beta) y(\theta)+\alpha(\theta)}{2 b}\right) \int_{\phi=\underline{\theta}}^{\phi=K(\theta)} G(\phi) \mathrm{d} \phi .
\end{aligned}
$$

Given assumptions (1) and (2), constraints (a) and (b) are equivalent to (c) $\Pi_{i}(\theta)=\int_{x=\theta}^{x=\bar{\theta}} q_{i}(x) \mathrm{d} x$. Thus, the regulator's problem is to select $\left(q_{i}^{s}(\theta), A^{s}(\theta), \alpha^{s}(\theta)\right)$ to maximize $\int_{\theta=\theta}^{\theta=\bar{\theta}} E_{\phi}(W(\theta, \phi)) f(\theta) \mathrm{d} \theta$ subject to constraint (c). Incorporating condition $[\overline{\mathbf{A}}]$ and (c) in the maximization exercise, we reduce the optimization problem to the following. Select $\left(q_{i}^{s}(\theta), A^{s}(\theta), \alpha^{s}(\theta)\right)$ to maximize $\int_{\theta=\underline{\theta}}^{\theta=\bar{\theta}} E_{\phi}\left(W^{s}(\theta, \phi)\right) f(\theta) \mathrm{d} \theta$, where

$$
\begin{aligned}
{[\tilde{\mathbf{A}}] \quad E_{\phi}\left(W^{s}(\theta, \phi)\right)=} & \left(a-z_{\beta}(\theta)\right) q_{i}(\theta)-\frac{b q_{i}^{2}(\theta)}{2}+y(\theta)\left(\frac{3 y(\theta)+2 \alpha(\theta)}{2 b}\right) G(K(\theta)) \\
& +P_{\beta}(\theta)-c .
\end{aligned}
$$

The optimization can now be done pointwise; that is, the regulator's problem now is to select $\left(q_{i}^{s}(\theta), A^{s}(\theta), \alpha^{s}(\theta)\right)$ that maximizes $E_{\phi}\left(W^{s}(\theta, \phi)\right)$ for each $\theta$. This problem is similar to that of the maximization problem in the proof of Proposition 1, where the regulator's problem was to select $\left(q_{i}^{f}(\theta), A^{f}(\theta), \alpha^{s}(\theta)\right)$ to maximize $E_{\phi}(W(\theta, \phi))$ given by $[\tilde{\mathbf{A}}]$. The only difference between the two is that, while in the former we had a term $-\theta q_{i}(\theta)$, in the latter this term is replaced by $-z_{\beta}(\theta) q_{i}(\theta)$. Thus, as long as we do not have any non-concavity problems, following steps similar to those in Proposition 1, we get the optimal quantity of the incumbent and the optimal access charges as $q_{i}^{s}(\theta)=q_{i}^{f}\left(z_{\beta}(\theta)\right), A^{s}(\theta)=A^{f}\left(z_{\beta}(\theta)\right)$ and $\alpha^{s}(\theta)=\alpha^{f}\left(z_{\beta}(\theta)\right)$ for each $\theta \in[\underline{\theta}, \bar{\theta}]$. It is important to note that, as in the known cost case, the entry ban solution under asymmetric information, i.e. $\left(q_{i}(\theta)=\left[a-z_{\beta}(\theta)\right] / b, A(\theta)=\frac{4}{b}\left(z_{\beta}(\theta)-\underline{\theta}\right)^{2}, \alpha(\theta)=-3\left(z_{\beta}(\theta)-\underline{\theta}\right)\right)$, is pointwise suboptimal, since for each type $\theta$ it yields a welfare not higher than the optimal solution. Substituting $\left(q_{i}^{s}(\theta), A^{s}(\theta), \alpha^{s}(\theta)\right)$ in (3) and then setting $\Pi_{i}(\theta)=\int_{x=\theta}^{x=\bar{\theta}} q_{i}(x) \mathrm{d} x$, we get $t^{s}(\theta)=t^{f}\left(z_{\beta}(\theta)\right)-(1-\beta)$ $L(\theta) q_{i}^{s}(\theta)+\int_{x=\theta}^{x=\bar{\theta}} q_{i}^{s}(x) \mathrm{d} x$.

If the distribution $f(\theta)$ is such that $z(\bar{\theta}) \leqslant \underline{\theta}+(11-6 \beta)^{2} \Delta / 2(56-32 \beta)$, then $H_{\beta}(z(\theta))$ is defined for all $\theta \in[\underline{\theta}, \bar{\theta}]$ and the solution given above is valid. If, however, $z_{\beta}(\theta)>\underline{\theta}+(11-6 \beta)^{2} \Delta / 2(56-32 \beta)$, we have an existence problem. This means that $H_{\beta}(z(\theta))$ is not defined when $z_{\beta}(\theta)$ is above $\underline{\theta}+$ $(11-6 \beta)^{2} \Delta / 2(56-32 \beta)$. Given that $z_{\beta}(\theta)$ is increasing in $\theta$, there is an existence problem for all $\theta \in[\tilde{\theta}(\beta), \bar{\theta}]$ where $\tilde{\theta}(\beta)=z_{\beta}^{-1}\left[\underline{\theta}+(11-6 \beta)^{2} \Delta / 2(56-32 \beta)\right]$. The existence problem comes from the non-concavity of the $E_{\phi}\left(W^{s}(\theta, \phi)\right)$ function for $\theta$ such that $z_{\beta}(\theta)>\underline{\theta}+(11-6 \beta)^{2} \Delta / 2(56-32 \beta)$. We incorporate the optimal values of $\left(\alpha^{s}(\theta), A^{s}(\theta)\right)$ in $E_{\phi}\left(W^{s}(\theta, \phi)\right)$, to get

$$
\begin{aligned}
E_{\phi}\left(W^{s}(\theta, \phi)\right)= & (a-z(\theta)) q_{i}(\theta) \\
& -\frac{b\left\{q_{i}(\theta)\right\}^{2}}{2}+\frac{4(1-\beta)^{2}\left(77-16 \beta-28 \beta^{2}\right)\left(P\left(q_{i}(\theta)\right)-\underline{\theta}\right)^{3}}{3 b \Delta(11-6 \beta)^{3}}-c .
\end{aligned}
$$

(C) The London School of Economics and Political Science 2007 
The second derivative of $E_{\phi}\left(W^{s}(\theta, \phi)\right)$ with respect to $q_{i}(\theta)$ gives

$$
\frac{\partial^{2} E_{\phi}\left(W^{s}(\theta, \phi)\right)}{\partial q_{i}(\theta)^{2}}=-b\left\{1-\left[\frac{8(1-\beta)^{2}\left(77-16 \beta-28 \beta^{2}\right)\left[D_{\beta}(\underline{\theta})-D_{\beta}(\theta)\right]}{(11-6 \beta)^{3}(56-32 \beta) \Delta}\right]\right\}
$$

The second derivative in condition (13) is negative only if $z_{\beta}(\theta)<\underline{\theta}+(11-6 \beta)^{2} \Delta / 2(56-32 \beta)$ and is not defined otherwise. Hence if the distribution $f(\theta)$ is such that (13) is not defined, the above solution is not valid for all $\theta \in[\tilde{\theta}(\beta), \bar{\theta}]$.

To solve this non-existence problem with pointwise optimization, we incorporate the optimum bunching procedure. For that we need to apply optimal control theory. We break the regulator's problem into two sub-problems. We define an interval $[\underline{\theta}, \tilde{\theta}(\beta))$, where we have full separability of types, and the interval $[\tilde{\theta}(\beta), \bar{\theta}]$, where we have pooling of types and $\tilde{\theta}(\beta)$ is an as-yet undetermined, cut-off point. The incentive compatibility problem in terms of the first derivative (i.e. $\left.\partial \Pi_{i}(\theta) / \partial \theta=-q_{i}(\theta)\right)$ acts as the equation of motion in the two sub-problems, and the condition $\Pi_{i}(\bar{\theta})=0$ (obtained from participation constraint) is the transversality condition. We solve for the two sub-problems and finally select the optimal cut-off point $\tilde{\theta}(\beta)$. This cut-off point turns out to be a point where we had existence problem with pointwise maximization. Thus, the optimal solution is identical to the pointwise optimization problem in the well defined zone and is a pooling one for higher types. Finally, as in Proposition 1, it is quite easy to verify that this solution dominates the entry ban solution.

\section{Proof of Proposition 5}

If $\tilde{\theta}(\beta)=\bar{\theta}$, then the entry limit is

$$
K^{s}(\theta)=K^{f}\left(z_{\beta}(\theta)\right)=\left(\frac{r_{2}(\beta)-1}{r_{1}(\beta)+r_{2}(\beta)}\right) H_{\beta}\left(z_{\beta}(\theta)\right)+\left(\frac{r_{1}(\beta)+1}{r_{1}(\beta)+r_{2}(\beta)}\right) \underline{\theta} .
$$

Thus, $K^{s}(\underline{\theta})=K^{f}\left(z_{\beta}(\underline{\theta})\right)=K^{f}(\underline{\theta})=\underline{\theta}$ since $z_{\beta}(\underline{\theta})=\underline{\theta}$. Here

$$
\begin{aligned}
& K^{s}(\bar{\theta}) \\
& \quad=\underline{\theta}+\frac{(1-\beta)\left\{\left(r_{1}(\beta)+r_{2}(\beta)\right) \Delta-\sqrt{\left(r_{1}(\beta)+r_{2}(\beta)\right)^{2} \Delta^{2}-16\left(r_{1}(\beta)+1\right) \Delta\left(z_{\beta}(\bar{\theta})-\bar{\theta}\right)}\right\}}{2\left(r_{1}(\beta)+1\right)} \\
& \quad<\bar{\theta} .
\end{aligned}
$$

The last inequality follows because (a) given $\tilde{\theta}(\beta)=\bar{\theta}$, the term inside the square root is nonnegative and

$$
(b)(1-\beta) \frac{\left(r_{1}(\beta)+r_{2}(\beta)\right)}{2\left(r_{1}(\beta)+1\right)}=(1-\beta) \frac{(11-6 \beta)}{(14-8 \beta)}<1 .
$$

Moreover, since

$$
K^{s}(\theta)=K^{f}\left(z_{\beta}(\theta)\right)
$$

and

$$
K^{f}\left(z_{\beta}(\theta)\right)
$$

and $z_{\beta}(\theta)$ are increasing in $z_{\beta}(\theta)$ and $\theta$ respectively, it follows that $K^{s}(\theta)$ is increasing in $\theta \in[\underline{\theta}, \bar{\theta}]$. Note that the rate of increase in $K^{s}(\theta)$ is ambiguous. Finally, given $z_{\beta}(\theta)>\theta$ for all $\theta \in(\underline{\theta}, \bar{\theta}]$, we get

$$
K^{s}(\theta)-K^{f}(\theta)=\frac{4(1-\beta)}{\left(r_{1}(\beta)+r_{2}(\beta)\right)}\left(H_{\beta}\left(z_{\beta}(\theta)\right)-H_{\beta}(\theta)\right)>0 \quad \text { for all } \quad \theta \in[\underline{\theta}, \bar{\theta}] .
$$


If $\tilde{\theta}(\beta)<\bar{\theta}$, then as in the previous case $K^{s}(\underline{\theta})=\underline{\theta}$. At point $\tilde{\theta}(\beta)$,

$$
H_{\beta}\left(z_{\beta}(\tilde{\theta}(\beta))\right)=\underline{\theta}+\frac{\left(r_{1}(\beta)+r_{2}(\beta)\right)^{2}}{8\left(r_{1}(\beta)+1\right)} \Delta .
$$

So

$$
K^{S}(\tilde{\theta}(\beta))=\underline{\theta}+\frac{(1-\beta)\left(r_{1}(\beta)+r_{2}(\beta)\right)}{2\left(r_{1}(\beta)+1\right)} \Delta<\bar{\theta} .
$$

Moreover,

$$
\forall \theta \in(\underline{\theta}, \tilde{\theta}(\beta)), K^{s}(\theta)=K^{f}\left(z_{\beta}(\theta)\right) \quad \text { and } \quad K^{f}\left(z_{\beta}(\theta)\right)
$$

and $z_{\beta}(\theta)$ are increasing in $z_{\beta}(\theta)$ and $\theta$ respectively. Therefore, $K^{s}(\theta)$ is increasing in $\theta \in(\underline{\theta}, \tilde{\theta}(\beta))$. To prove the final step, observe first that $\tilde{\theta}(\beta)<\bar{\theta} \Rightarrow \tilde{\theta}(\beta)<\bar{\theta}(\beta) \leqslant \bar{\theta}$ since $z_{\beta}(\theta)>\theta$ for all $\theta \in(\underline{\theta}, \bar{\theta}]$. Also observe that

$$
H_{\beta}\left(z_{\beta}(\tilde{\theta}(\beta))\right)=H_{\beta}(\bar{\theta}(\beta))=\underline{\theta}+\frac{\left(r_{1}(\beta)+r_{2}(\beta)\right)^{2}}{8\left(r_{1}(\beta)+1\right)} \Delta .
$$

Using these two observations we get

(a) $K^{s}(\theta)-K^{f}(\theta)=\frac{4(1-\beta)}{\left(r_{1}(\beta)+r_{2}(\beta)\right)}\left\{H_{\beta}\left(z_{\beta}(\theta)\right)-H_{\beta}(\theta)\right\}>0$ for all $\theta \in(\underline{\theta}, \tilde{\theta}(\beta)]$,

(b) $K^{s}(\theta)-K^{f}(\theta)=\frac{4(1-\beta)}{\left(r_{1}(\beta)+r_{2}(\beta)\right)}\left\{H_{\beta}\left(z_{\beta}(\tilde{\theta}(\beta))\right)-H_{\beta}(\theta)\right\}>0 \quad$ for all $\theta \in(\tilde{\theta}(\beta), \bar{\theta}(\beta))$

(c) $K^{s}(\theta)-K^{f}(\theta)=\frac{4(1-\beta)}{\left(r_{1}(\beta)+r_{2}(\beta)\right)}\left\{H_{\beta}\left(z_{\beta}(\tilde{\theta}(\beta))\right)-H_{\beta}(\bar{\theta}(\beta))\right\}=0 \quad$ for all $\theta \in[\bar{\theta}(\beta), \bar{\theta}]$.

From (a), (b) and (c), it follows that $K^{f}(\theta) \leqslant K^{s}(\theta)$ for all $\theta \in(\underline{\theta}, \bar{\theta}]$.

\section{ACKNOWLEDGMENTS}

We gratefully acknowledge financial support from the Brussels Capital Region and from the Deutsche Forschungsgemeinschaft Graduiertenkolleg (DFG) 629 at the University of Bonn. We are thankful to seminar and conference participants at the University of Bonn, the EARIE meeting held in Helsinki and the First Conference on 'Railroad Industry Structure, Competition and Investment' in Toulouse, France.

\section{NOTES}

1. This monopoly bottleneck does not exist if, owing to technical changes, the entrant(s) can bypass the infrastructure of the monopolist. For example, it is now possible to provide telecommunication services with wireless or cable TV networks, and these companies can compete with wire line networks on the telecommunication services market. However, when this sort of bypass is not possible we have the monopoly bottleneck problem.

2. The European Commission specifies that access fees and access conditions to the bottleneck input should be designed on a non-discriminatory basis. For example, in the preamble to the rail directive 2002/14/EC, it is required that: 'To ensure transparency and non-discriminatory access to rail infrastructure for all railway undertakings, all the necessary information required to use the access rights are to be published in a network statement', and 'The charging and capacity allocation schemes should permit equal and non-discriminatory access for all undertakings.' Non-discriminatory access to the railway tracks has been advocated by a large number of competition authorities, at least for freight operators. For passenger services, access is limited to moderate competition (in the UK for example) (see Campos and Cantos 1999). Similarly, in telecommunications, non-discriminatory access of wire lines to competitors is recommended.

3. Here $\mathbf{R}_{+}$represents the non-negative orthant of the real line.

(C) The London School of Economics and Political Science 2007 
4. In our model, if the regulator applies the ECPR, then entry is efficient but not the market structure. See the proof of Proposition 3 in the Appendix.

5. In a standard regulation model with an exogenously given market structure and with linear demand, the surplus is a quadratic function of $q_{i}$. In this model, the entry depends on the regulated mechanism and the expected surplus is cubic in $q_{i}$. Hence the concavity of the problem must be checked.

6. It is worth noting that if the downstream market were more competitive, i.e. if there were a larger number of potential entrants, the entrants would have had less market power and hence the regulator would have subsidized them less. Being distortionary, the per-unit access charge is used only to cancel (partially) the entrant's market power. Conversely, the non-distortionary fixed fee is used by the regulator partially to transfer the profit of the unregulated competitor to the incumbent. This is because the incumbent owns the essential facility that needs financing. Again, if we had a single tariff (a Ramsey price with $A(\theta)=0$ ), instead of a two-part tariff, the per-unit fee would be positive. In this case, the access prices is used (i) to regulate entry, (ii) to raise money from the entrant and (iii) to influence the entrant's supply decisions.

7. If transfers are not possible (or are limited), the regulator should raise a larger contribution to the infrastructure financing from the firms. Since competition destroys profit, to increase the firms' contributions the regulator will deter more entry and increases the incumbent's price. Similarly, if there is a positive shadow cost of public funds, the regulator will restrict more entry to increase the firms' share in the infrastructure financing.

8. Caillaud (1990) demonstrates that, when a competitive fringe can compete with a regulated monopolist, in his case without using the monopolist's essential facility, the problem may not satisfy concavity, even if the virtual type function is increasing in type.

9. McAfee and McMillan (1987) have a similar result in the context of auctions.

10. The ECPR is a per-unit access charge that guarantees efficient entry on the downstream market. This access price is equal to the incumbent's opportunity cost, i.e., in our notation, $\alpha(\theta)=P\left(q_{i}(\theta)\right)-\theta$. See Armstrong (2001).

\section{REFERENCES}

Auriol, E. and LAFFOnT, J. J. (1993). Regulation by duopoly. Journal of Economics and Management Strategy, 1, 507-33.

Armstrong, M. (2001). Access pricing, bypass and universal service. American Economic Review, Papers and Proceedings, 91, 297-301.

BARON, D. and MYERSON, R. (1982). Regulating a monopolist with unknown costs. Econometrica, 50, 911-30.

Baumol, W., Panzar, J. and Willig, R. (1982). Contestable Markets and the Theory of Industry Structure. New York: Harcourt Brace.

Caillaud, B. (1990). Regulation, competition and asymmetric Information. Journal of Economic Theory, 52, $87-110$.

and Tirole, J. (2004). Essential facility financing and market structure. Journal of Public Economics, 88, 667-94.

CAmpos, J. and CAntos, P. (1999). Regulating privatized rail transport. Paper 2064, World Bank, Country Economics Department.

DANA, J. and SPIER, K. (1994). Designing a private industry: government auction with endogenous market structure. Journal of Public Economics, 53, 127-47.

De Fraja, G. (1999). Regulation and access pricing with asymmetric information. European Economic Review, 43, 109-34.

Gautier, A. and Mitra, M. (2006). Regulating a monopolist with limited funds. Economic Theory, Exposita Note, 27, 705-18.

GUESNERIE, R. and LAFFOnT, J. J. (1984). A complete solution to a class of principal-agent problems with an application to the control of a self-managed firm. Journal of Public Economics, 25, 329-69.

Jehiel, P. and Moldovanu, B. (2004). The design of an efficient private industry. Journal of the European Economic Association, Papers and Proceedings, 2-3, 516-25.

LAFFont, J. J. and Tirole, J. (1994). Access pricing and competition. European Economic Review, 38, $1673-710$. and (2000). Competition in Telecommunications. Cambridge, Mass., MIT Press.

LEWis, T. and SAPPINGTON, D. (1999). Access pricing with unregulated downstream competition. Information Economics and Policy, 11, 73-100.

McAfee, P. and McMillan, J. (1987). Auctions and bidding. Journal of Economic Literature, 25, $708-47$.

Mougeot, M. and NAegelen, F. (2005). Designing a market structure when firms compete for the right to serve the market. Journal of Industrial Economics, 53, 393-416.

World BANK (2002). World Development Report 2002: Building Institutions for Markets. Geneva: World Bank. 